\title{
Sequential measurements of procalcitonin levels in diagnosing ventilator-associated
} pneumonia

\author{
P. Ramirez*, M.A. Garcia\#, M. Ferrer", J. Aznar ${ }^{+}$, M. Valencia", J.M. Sahuquillo ${ }^{\S}$, \\ R. Menéndez ${ }^{f}$, M.A. Asenjo ${ }^{\# \#}$ and A. Torres ${ }^{\natural}$
}

ABSTRACT: The utility of procalcitonin levels to improve the accuracy of clinical and microbiological parameters in diagnosing ventilator-associated pneumonia (VAP) was evaluated.

Sequential measurement of procalcitonin and C-reactive protein levels and the calculation of the simplified Clinical Pulmonary Infection Scores (CPIS) were performed in 44 patients mechanically-ventilated for $>48 \mathrm{~h}$ with neither active infection for the duration or suspicion of VAP. Patients who developed extrapulmonary infection were excluded.

In total, 20 cases were suspected of having VAP and diagnosis was microbiologically confirmed in nine. In patients with confirmed VAP, procalcitonin levels were higher than in those without VAP. C-reactive protein levels and CPIS were lower in patients without suspected VAP, but could not discriminate confirmed and nonconfirmed suspicion of VAP. The best sensitivity and specificity (78 and 97\%, respectively) corresponded to procalcitonin. The CPIS resulted in the same sensitivity, but had a lower specificity (80\%). C-reactive protein had the worst sensitivity $(56 \%)$, but a good specificity $(91 \%)$. A CPIS $\geqslant 6$ combined with serum levels of procalcitonin $\geqslant 2.99 \mathrm{ng} \cdot \mathrm{mL}^{-1}$ did not improve the sensitivity $(67 \%)$, but resulted in $100 \%$ specificity.

Procalcitonin might be useful in the diagnosis of ventilator-associated pneumonia. Combined values of Clinical Pulmonary Infection Scores and procalcitonin below the cut-off points excluded false-positive diagnoses of ventilator-associated pneumonia.

KEYWORDS: C-reactive protein, procalcitonin, simplified Clinical Pulmonary Infection Scores, ventilator-associated pneumonia

V entilator-associated pneumonia (VAP) is an important problem in daily practice in intensive care medicine since it is associated with a more prolonged hospital stay and greater mortality $[1,2]$. However, the diagnosis of VAP continues to be difficult [3]. The classical clinical criteria; the appearance of infiltrates on chest radiographs, purulence of respiratory secretions, variations in body temperature or the number of circulating leukocytes, have a low specificity for the diagnosis of VAP. Microbiological confirmation takes $24-72 \mathrm{~h}$ and the frequent previous use of antibiotics may give false-negative information. The lack of consensus between different authors and the poor accuracy in the diagnostic criteria used, demonstrate the diversity between the values published and the incidence of VAP [1-3]. In this context, the identification of biological markers may aid in the diagnostic algorithm of VAP. One of these markers is procalcitonin, which is secreted by the organism as part of the systemic inflammatory response but only when triggered by infection [4]. The levels of procalcitonin have been successfully applied in different diseases in order to determine the presence of an active infectious process [5]. Nonetheless, few studies have analysed the behaviour of procalcitonin levels when the infection is in the lung [6-9]. Furthermore, there are even fewer studies aiming to establish a diagnostic cut-off value of procalcitonin levels in VAP and their results are discordant [10-12].

The hypothesis of the present study was that serum procalcitonin values may aid in the diagnosis of VAP. A cohort study was performed with sequential measurement of procalcitonin levels in patients receiving mechanical ventilation in the present authors' unit, with the aim of determining the utility of procalcitonin levels in the diagnosis of VAP and establishing a cut-off point, while attempting to eliminate all interference due to extrapulmonary infections.

\section{AFFILIATIONS}

*Intensive Care Unit,

Depts of ${ }^{+}$Biopathology,

${ }^{\S}$ Microbiology, and

${ }^{f}$ Pneumology, Universal Hospital La

$\mathrm{Fe}$, and

\#Intensive Care Unit, Sagunto

Hospital, Sagunto, Valencia,

\#\#Evaluation, Support and

Prevention Unit, and

-Intensive and Intermediate

Respiratory Care Unit, Pneumology

Service, Thorax Clinic Institute, Clinic

Hospital, Biomedical Research

Institute August Pi I Sunyer

(IDIBAPS), Barcelona, Spain.

CORRESPONDENCE

A. Torres Servei de Pneumologia,

Institut Clínic del Tòrax, Hospital

Clinic, Villarroel, 170. E-08036

Barcelona, Spain

Fax: 34932275549 E-mail:atorres@

ub.edu

Received:

July 112007

Accepted after revision:

October 072007

This study was supported by CIBERES (ISCiii-CB06/06/0028),

2005 Redes temáticas de

investigación cooperative. 00822, a

European Respiratory Society

Fellowship and IDIBAPS.A. Torres, M

Ferrer and R. Menédez are

Investigators from CIBERES 06/06/

0028.M. Valencia is a research fellow

in Hospital Clínic, Barcelona, Spain,

from Universidad Pontificia

Bolivariana de Medellín, Colombia,

supported by Fondo de

Investigaciones Sanitarias

Fellowship.

STATEMENT OF INTEREST

None declared.

European Respiratory Journal

Print ISSN 0903-1936

Online ISSN 1399-3003 


\section{METHODS}

\section{Design and inclusion criteria}

A prospective, observational study was performed in the intensive care unit (ICU) of a tertiary university teaching hospital between September, 2004 and February, 2006. The ICU has 21 beds and attends medical patients. The present study was approved by the ethical committee of the hospital and informed consent was obtained from the families of the patients studied.

Patients receiving mechanical ventilation expected to continue $>48 \mathrm{~h}$ were included. Exclusion criteria were active infection at admission and previous diagnosis of small cell lung cancer or medullar cancer of the thyroid. Patients developing nosocomial infection other than VAP during hospitalisation were excluded upon diagnosis of these infections.

\section{Data collection protocol}

The following data were collected on study inclusion: sex; comorbidities; severity scores prior to intubation, including the Glasgow Coma Scale, the Acute Physiology Score (APS), the Acute Physiology And Chronic Health Evaluation (APACHE) score-II [14], the Sepsis-related Organ Failure Assessment (SOFA); and the simplified Clinical Pulmonary Infection Score (CPIS) [15]; the presence or not of the Systemic Inflammatory Response Syndrome (SIRS) [16]; the causes of the initiation of mechanical ventilation; and the use of antibiotics the week prior to inclusion in the study.

Daily evaluation of the patients included: calculation of the severity scores (APS, APACHE-II and SOFA); evaluation of SIRS; calculation of the simplified CPIS; evaluation of the clinical diagnostic criteria of VAP; presence of other infections [17]; and use of systemic antibiotics.

On the day of study inclusion, procalcitonin and C-reactive protein (CRP) levels were determined. These determinations were repeated every $48 \mathrm{~h}$ until the end of the study.

In cases with clinical suspicion of VAP, samples were collected in order to determine procalcitonin and CRP levels in serum and in bronchoalveolar lavage (BAL). These evaluations were repeated $72 \mathrm{~h}$ after case confirmation.

The follow-up of patients included in the study continued until the end of mechanical ventilation or until the appearance of the first episode of suspicion of VAP.

\section{Definitions}

Suspicion of VAP was established if patients met one of the following. 1) Classical clinical criteria: the appearance of a new pulmonary infiltrate on chest radiograph or progression of an existing infiltrate, together with two of the following criteria: temperature $>38^{\circ} \mathrm{C}$; leukocytosis $>12,000 \cdot \mathrm{mm}^{-3}$; or purulent respiratory secretions [18]. 2) Simplified CPIS score $>5$ points [15].

The confirmation of VAP was defined by the quantitative culture of BAL with $\geqslant 10^{4}$ colony forming units (CFU) $\cdot \mathrm{mL}^{-1}$ [19] of a potentially pathogenic microorganism. At this time, blood and urine were also collected [20] in order to eliminate the presence of other nosocomial infections [17]. Patients were classified into three groups based upon the evolution of the appearance of VAP: 1) without suspicion of VAP and absence of pulmonary infiltrates; 2) with nonconfirmed VAP (with initial suspicion of VAP not confirmed microbiologically); and 3) with VAP confirmed microbiologically. An effort was made to find an alternative diagnosis in those patients from the second group.

\section{Evaluation of the evolution of VAP}

Cases confirmed with VAP were evaluated at $72 \mathrm{~h}$. Nonresponders were those cases in which at least one of the following criteria were present: 1) absence of improvement in arterial oxygen tension $\left(\mathrm{Pa}, \mathrm{O}_{2}\right)$ / inspiratory oxygen fraction $\left.\left(\mathrm{FI}, \mathrm{O}_{2}\right) ; 2\right)$ persistence of fever $\left(\geqslant 38^{\circ} \mathrm{C}\right)$ together with purulent respiratory secretions; 3 ) increase in the respiratory infiltrate on chest radiograph of $>50 \%$; or 4 ) the development of septic shock or multiorgan failure [21].

\section{Study of inflammatory markers}

The blood samples taken for determination of inflammatory markers were centrifuged $(75 \times \mathrm{g}, 10 \mathrm{~min})$ and the supernatant was frozen at $-20^{\circ} \mathrm{C}$. Procalcitonin was measured by TimeResolved Amplified Cryptate Emission technology in a Kryptor analyser (Brahms Diagnostica, Berlin, Germany). Measurement of CRP was performed with an immunoturbidimetric method using a commercial kit (Tina-quant CRP; Roche Diagnostics, Mannheim, Germany).

\section{Statistical analysis}

Categorical variables were compared using Chi-squared and Fisher's exact tests when appropriate. Comparison of numerical and categorical variables was performed with unpaired t-tests or Mann-Whitney U-tests when the latter were dichotomic, and ANOVA or Kruskal-Wallis H-tests were carried out for variables with more than two categories. The sensitivity, specificity and positive and negative predictive values of CPIS and procalcitonin and CRP levels were determined by comparing patients with confirmed VAP and those without pneumonia (nonconfirmed suspicion and no suspicion). Receiver operating characteristic (ROC) curves were created to determine the optimal cut-off values and to evaluate the general discriminative capacity of these indices. The optimal cut-off values were obtained from the best sensitivity/specificity ratios. Results were expressed as median with interquartile $(25-75 \%)$ range.

\section{RESULTS}

\section{Description of the population}

All patients receiving mechanical ventilation were screened during the study period (fig. 1). A total of 52 patients were included in the study. Eight of the patients initially included were later excluded as in five cases, mechanical ventilation was used for $<48 \mathrm{~h}$ and, in three cases, active infection was diagnosed after the time of inclusion. No patient developed nosocomial infection other than VAP.

The reason for admission to the ICU was: cerebral vascular accident in 30 (68\%) cases; cardio-respiratory failure followed by reanimation in nine $(20.5 \%)$ cases; coma of another aetiology in two $(4.5 \%)$ cases; one acute respiratory failure of neuromuscular origin; one cardiogenic shock secondary to acute myocardial infarction; and one exacerbation of chronic respiratory insufficiency. The most common comorbidities were diabetes mellitus $(20.5 \%)$, smoking $(11.4 \%)$, systemic steroids $(4.5 \%)$ and chronic obstructive pulmonary disease $(2.3 \%)$. 


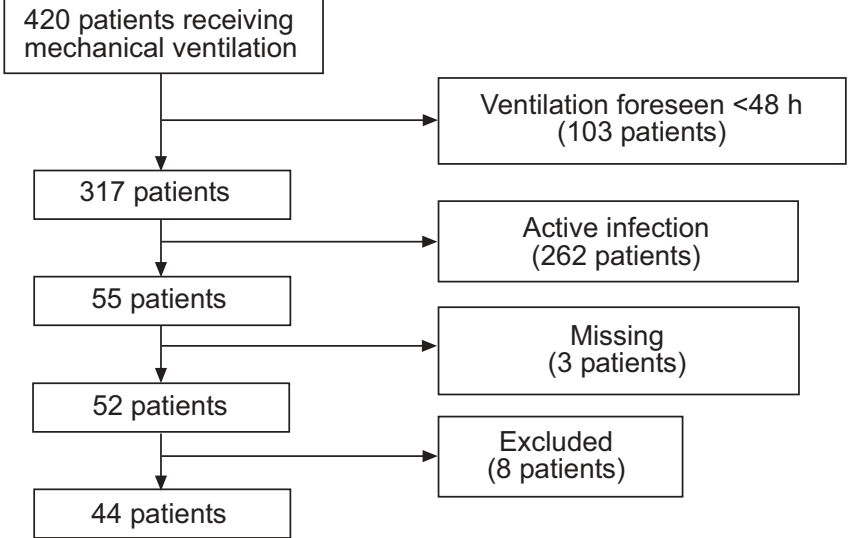

FIGURE 1. Screening of patients undergoing mechanical ventilation during the study period.

In total 20 patients were suspected of having VAP throughout their stay in the ICU. Microbiological analysis of the BAL confirmed the presence of VAP in nine cases. The causative microorganisms of the confirmed cases of VAP were: Staphylococcus aureus in three cases; Acinetobacter baumanii in three cases; Klebsiella pneumoniae in two cases and Streptococcus pneumoniae in one case. After $72 \mathrm{~h}$ of evolution, two patients were classified as responders and four as nonresponders, while the remaining three could not be evaluated due to death as a consequence of brain injury before $72 \mathrm{~h}$.

The non-microbiologically confirmed cases $(n=11)$ were not considered as having VAP. Only in three of these patients was there a positive culture of BAL, all with $<10^{3} \mathrm{CFU} \cdot \mathrm{mL}^{-1}$ of $A$. baumanii, coagulase-negative Staphylococcus species and Haemophilus influenzae. In $18 \%$ of these patients an antibiotic treatment was in use.

Table 1 shows the characteristics of the patients at the time of study inclusion. No statistically significant differences were found in any of the variables studied.

Table 2 describes the characteristics of the three groups the day on which VAP was suspected. In patients not suspected of having VAP the characteristics of day 4 were used (median of day of evolution in which VAP was suspected in the other two groups). The administration of systemic antibiotic treatment on the days prior to suspicion of VAP was similar in the three groups. No differences were found in regard to the presence of SIRS, APACHE-II, APS, temperature and leukocyte count. In the groups with confirmed and nonconfirmed suspicion of VAP, the SOFA score and simplified CPIS scale were greater and the $\mathrm{PaO}_{2} / \mathrm{FIO}_{2}$ ratio was lower than the group without clinical suspicion of VAP.

\section{Results of C-reactive protein and procalcitonin in the diagnosis of VAP}

The differences in the plasma levels of procalcitonin and CRP among the three groups the day of VAP suspicion were statistically significant, especially in regard to procalcitonin $(\mathrm{p}<0.001)$, with the highest values corresponding to patients with microbiological confirmation of VAP (table 2). When comparing patients with VAP and nonconfirmed VAP, only procalcitonin levels were significantly higher in patients with

TABLE 1 Characteristics of the patients on the day of study inclusion at start of mechanical ventilation

VAP

Nonconfirmed

Males $\mathrm{n}$

ICU stay days

Hospitalisation days

GCS prior to intubation

Previous antibiotics days

Presence SIRS

APACHE-II

APS

SOFA

CPIS

Temperature ${ }^{\circ} \mathrm{C}$

Leukocytes $10^{9}$ cells $\cdot \mathrm{L}^{-1}$

$\mathrm{Pa}, \mathrm{O}_{2} / \mathrm{Fl}_{1} \mathrm{O}_{2} \mathrm{mmHg}$

CRP $\mathrm{mg}^{\mathrm{dL}} \mathrm{dL}^{-1}$

Procalcitonin $\mathrm{ng} \cdot \mathrm{mL}^{-1}$

$$
\begin{gathered}
63(52-72) \\
16 \\
10(5.5-16.5) \\
11.5(6.5-25) \\
6(3-8) \\
0 \\
19(79) \\
20(16.5-24) \\
14.5(11.5-16.5) \\
6(5-8) \\
3(1.5-3) \\
37.5(36.3-37.9) \\
11.1(8.3-15.5) \\
298(229-349) \\
3.9(1.5-6.4) \\
0.15(0.10-0.73)
\end{gathered}
$$

11
$66(53-73)$
5
$16(10-18.5)$
$16(10-25.5)$
$6(3-7.5)$
0
$9(82)$
$18(15.5-23)$
$13(11-19)$
$6(5.5-7.5)$
$2(1.5-4)$
$37.0(36.0-38.5)$
$11.1(7.6-13.5)$
$290(255-382)$
$4.3(1.2-6.4)$
$0.33(0.17-1.90)$

$66(53-73)$

5

$0.33(0.17-1.90)$ p-value

Data are presented as median (interquartile range) and n (\%), unless otherwise stated. VAP: ventilator-associated pneumonia; ICU: intensive care unit; GCS: Glasgow Coma Score; SIRS: Systemic Inflammatory Response Syndrome; APACHE: Acute Physiology And Chronic Health Evaluation score; APS: Acute Physiology Score; SOFA: Sepsis-related Organ Failure Assessment; CPIS: Clinical Pulmonary Infection Score; $\mathrm{Pa}, \mathrm{O}_{2} / \mathrm{Fl}_{1} \mathrm{O}_{2}$ : arterial oxygen tension / inspiratory oxygen fraction; CRP: C-reactive protein. $1 \mathrm{mmHg}=0.133 \mathrm{kPa}$ 
TABLE 2 Characteristics of patients on day of ventilator-associated pneumonia (VAP) suspicion

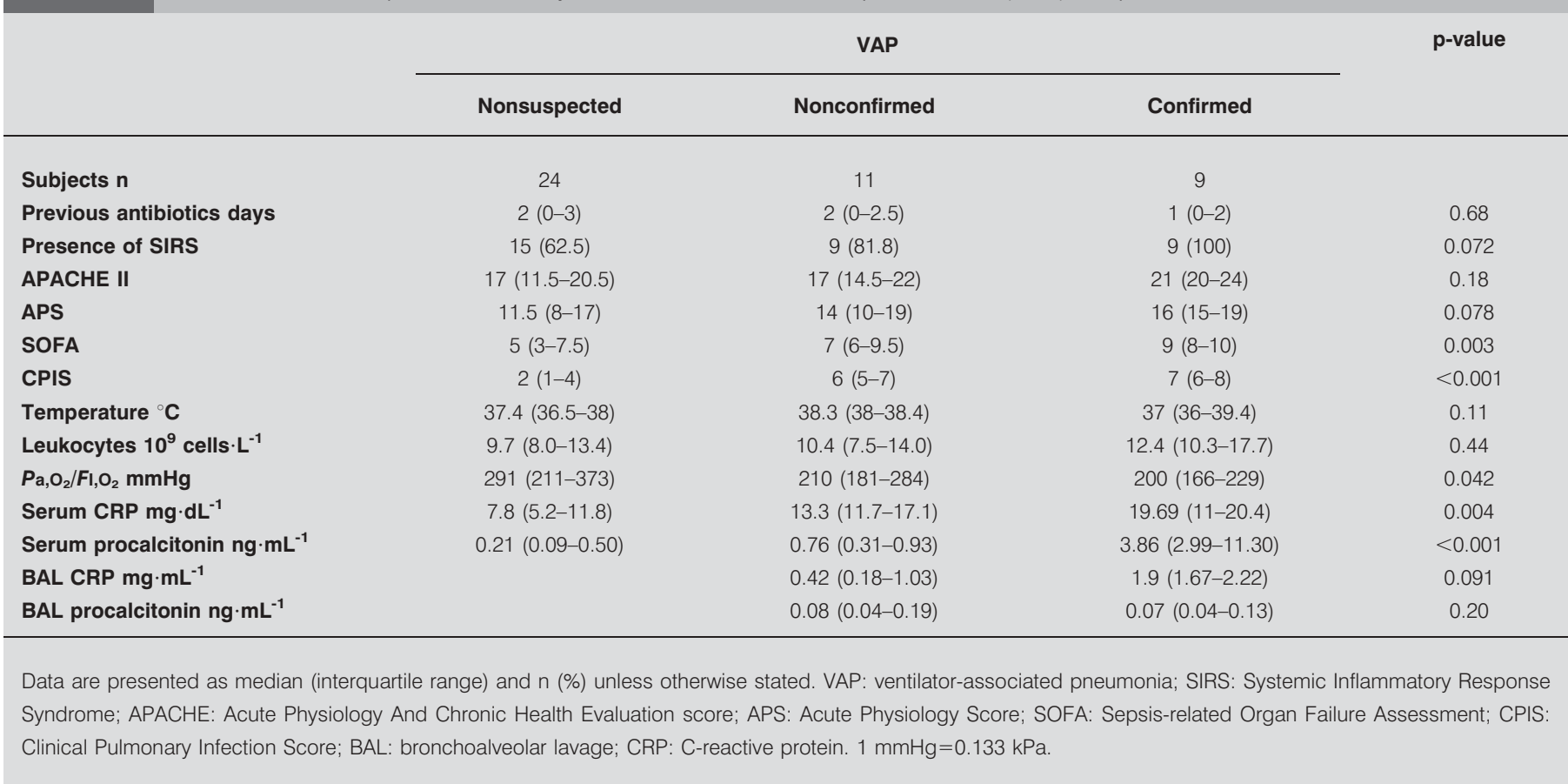

VAP $(p=0.001)$, while CRP levels were not significantly different.

Analysis of procalcitonin levels in BAL did not show differences between the group with VAP and the group with nonconfirmed VAP (table 2). A nonsignificant trend to higher BAL levels of CRP was shown in patients with confirmed VAP $(\mathrm{p}=0.091)$.

Diagnostic value of serum levels of procalcitonin and CRP and the CPIS scale

Table 3 shows the optimal cut-off values of procalcitonin and CRP levels and simplified CPIS used to calculate the operative

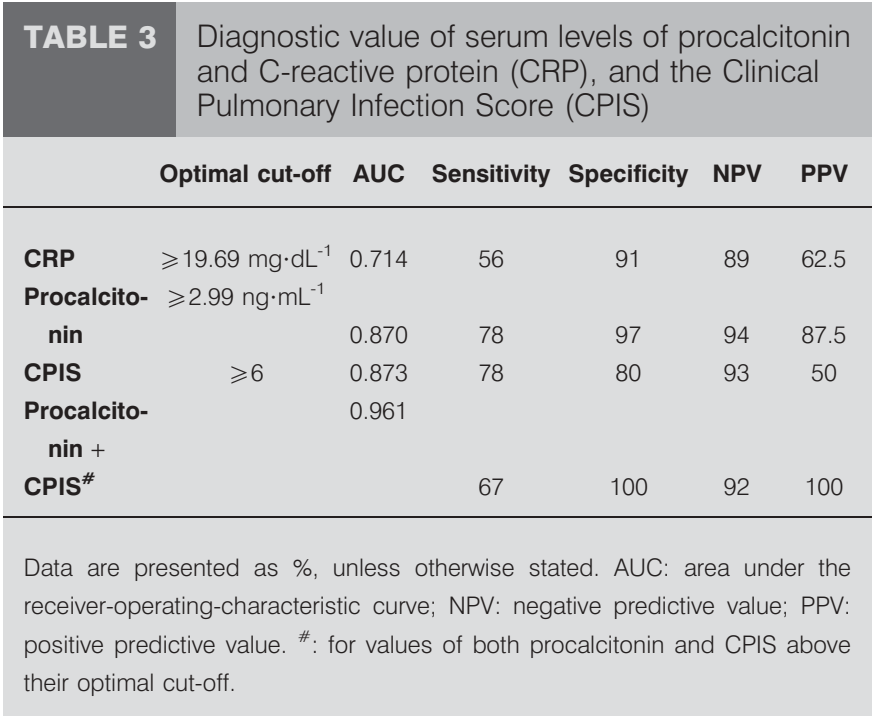

indices, as well as the general discriminative capacity of these indices to differentiate between the presence and the absence of VAP, assessed by the area under the ROC curve (fig. 2). The best sensitivity and specificity (78 and $97 \%$, respectively) corresponds to procalcitonin. The simplified CPIS resulted in the same sensitivity, but had a lower specificity $(80 \%)$. Creactive protein had the worse sensitivity $(56 \%)$, but a good specificity $(91 \%)$.

The use of simplified CPIS $\geqslant 5$ points in combination with serum levels of procalcitonin $\geqslant 2.99 \mathrm{ng} \cdot \mathrm{mL}^{-1}$ did not improve the sensitivity $(67 \%)$ but resulted in $100 \%$ specificity.

The diagnostic capacity of procalcitonin, CRP and the simplified CPIS score were maintained upon comparing all patients with clinical suspicion of VAP (with and without microbiological confirmation) versus the group without suspicion of VAP. The areas under the ROC curves were $0.870,0.714$ and 0.873 for procalcitonin, CRP and the simplified CPIS, respectively. However, on comparing the group with confirmed VAP versus the group with nonconfirmed VAP, the discriminative capacity only remained with procalcitonin (the area under the ROC curve was 0.828) while in the other variables, the area under the ROC curve fell to 0.544 and 0.651 for CRP and the simplified CPIS, respectively.

\section{DISCUSSION}

The results of the present study show that the serum level of procalcitonin is useful in the diagnosis of VAP. The simplified CPIS scale has a similar sensitivity but lower specificity than the serum level of procalcitonin. Combining the clinical data, assessed by the CPIS scale, and serum procalcitonin levels excluded false-positive diagnoses of VAP in the present population. 

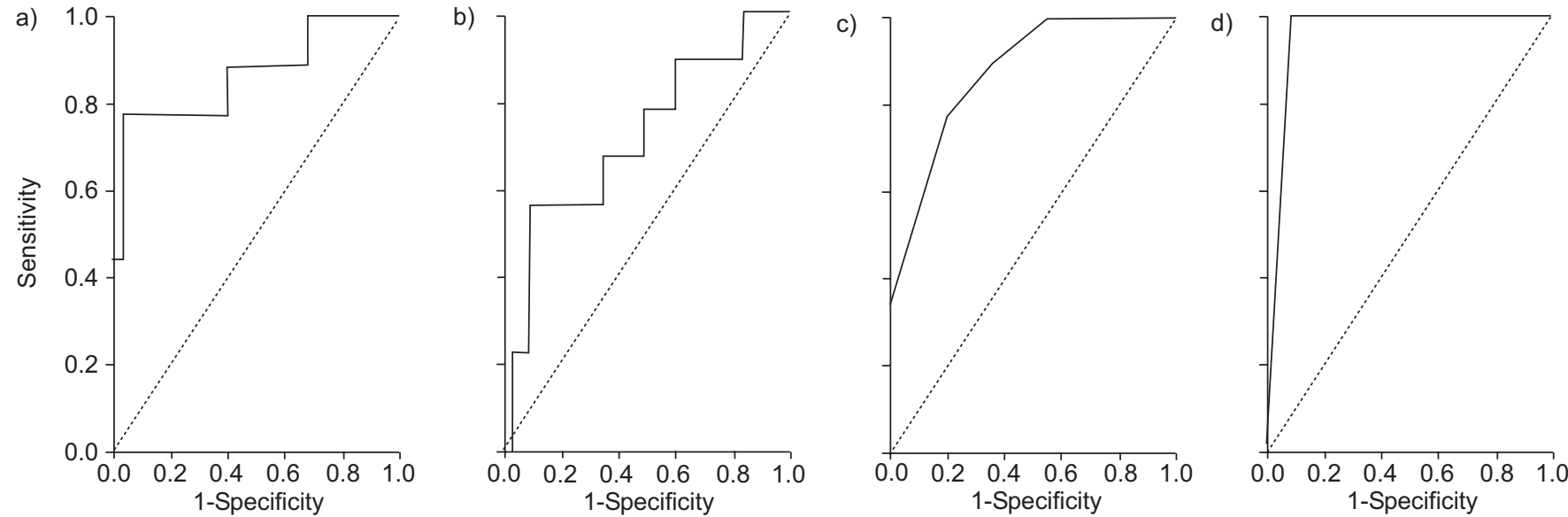

FIGURE 2. Receiver-operating-characteristic (ROC) curves for a) procalcitonin (area under ROC curve (AUC): 0.870; 95\% confidence interval (CI): 0.712-1.027), b) Creactive protein (AUC: $0.714 ; 95 \%$ Cl: $0.515-0.914$ ), c) the simplified Clinical Pulmonary Infection Score (CPIS; AUC: $0.873 ; 95 \%$ Cl: $0.757-0.989$ ) and d) the combination of procalcitonin+CPIS (AUC: 0.961; 95\% Cl: 0.905-1.016).

Currently, the diagnosis of VAP is still established by the presence of some clinical criteria together with a positive quantitative culture of a representative respiratory sample. Nonetheless, the incidence of VAP in patients receiving mechanical ventilation varies widely from one study to another (9-27\%). This discordance may lie in the lack of consensus regarding the diagnostic criteria which, in turn, is due to the inaccuracy of the criteria used. The sensitivity and specificity of the classical clinical criteria are deficient. One study in post mortem pulmonary biopsies [3] reported that the combination of new and persistent infiltrates on chest radiographs and two or three of: fever $\left(>38^{\circ} \mathrm{C}\right)$, leukocytes $(>12,000$ cells $\cdot \mathrm{mm}^{-3}$ ) or purulent tracheal secretion, had a sensitivity of $69 \%$ and a specificity of $75 \%$. With regard to microbiological confirmation, the problems are the delay in obtaining culture results (48-72 h), the variability of the cut-off value of CFU used and the potential interference of antibiotic use. In this context, the determination of biological markers, such as procalcitonin and CRP levels and soluble triggering receptor expressed on myeloid cells, might be of help in the diagnostic process of VAP $[12,13]$.

Procalcitonin is secreted as part of the systemic inflammatory response to infection. Serum values of procalcitonin vary greatly based on the type and severity of infection [4, 5]. Few studies have analysed the behaviour of procalcitonin in lung infection [6-9]. However, the use of procalcitonin levels for the diagnosis of VAP has not been established.

In the present study, the relationship between procalcitonin levels and VAP has been evaluated and a diagnostic cut-off point of $2.99 \mathrm{ng} \cdot \mathrm{mL}^{-1}$, which provides the best sensitivity and specificity, has been established. This finding is of particular importance in order to distinguish infectious from noninfectious aetiologies in patients with pulmonary infiltrates, which is a current dilemma in the clinical practice. The differences in the cut-off point of procalcitonin levels found in other diseases (e.g. community-acquired pneumonia) could be explained by the increased and complex inflammatory response in critically ill mechanically ventilated patients.
The results presented herein are different from those obtained by OPPERT et al. [10] (cut-off point $1 \mathrm{ng} \cdot \mathrm{mL}^{-1}$ ). However, it seems that the diagnostic criteria of VAP used by these authors differed greatly from those currently accepted. DufLo et al. [11] found a diagnostic cut-off point of $3.9 \mathrm{ng} \cdot \mathrm{mL}^{-1}$, with a sensitivity of $41 \%$ and a specificity of $100 \%$; however, they only included patients with suspicion of VAP and they did not control for extrapulmonary infections, carried out in the present study. Similar to the study by Duflo et al. [11], no differences were found in alveolar procalcitonin values among different groups. Procalcitonin mainly forms part of the systemic response to infection and, consequently, alveolar values are lower than serum values.

GIBOT et al. [12] analysed the behaviour of procalcitonin in pneumonia (community- and ventilator-acquired) and concluded that there were no differences between the groups with and without pneumonia. However, a high percentage of patients with pneumonia had other infections, which increased the serum procalcitonin values.

The results of the present study indicate that, although CRP levels may have a certain utility in the diagnosis of VAP, procalcitonin was better as a biological marker of bacterial infection. In a previous meta-analysis performed by SIMON et al. [5], procalcitonin was also superior to CRP in different types of bacterial infections. PóvoA et al. [22] recently studied the role of CRP to detect infections in critically ill patients. In that study, CRP was a reasonable marker of bacterial infection when combined with body temperature. However, the groups studied were very heterogeneous and therefore conclusions for specific groups have to be made cautiously, since CRP values are not only influenced by infections but also by any pro-inflammatory circumstance of noninfectious nature $[22,23]$.

The simplified CPIS scale, as used by LuNA et al. [15], has proven to be useful in the diagnosis and prognosis of VAP, and the results presented herein are in agreement with previous publications. In that study, $100 \%$ of patients with VAP had a 
score $>5$ points, as was observed in patients with confirmed VAP taking part in the present study. Combining both the simplified CPIS and procalcitonin levels for the initial diagnosis of VAP, a 100\% specificity was obtained. The major advantage of this combination is the avoidance of falsepositive results. This can be very useful in order to restrict unnecessary antibiotic treatments. However, the risk of undertreatment has to be taken into account.

By contrast, in a recent preliminary report [24], crude levels of procalcitonin did not result in good diagnostic accuracy for VAP. The explanation for this is the population was selected without avoiding extrapulmonary infections.

Procalcitonin is a good prognostic marker in patients with VAP, as has been demonstrated by LUYT et al. [25]. Due to the small number of responders and nonresponders after $72 \mathrm{~h}$ of evolution in the present study, the utility of procalcitonin levels for the assessment of antibiotic treatment response could not be evaluated.

The main limitations of the present study are the small sample size and the selection of the type of patients. These limitations are the consequence of avoiding the multiple confounding factors usually present in critically ill patients for this type of study. In order to avoid the interferences of extrapulmonary infections in the determination of procalcitonin values, it was necessary to exclude patients who had active infection at the time of initiating mechanical ventilation. Similarly, patients who developed extrapulmonary infection during follow-up were excluded. Therefore, the variations observed in the plasma procalcitonin values were only due to the presence or absence of VAP. Another limitation is that, despite the use of quantitative microbiology to confirm VAP, the potential falsepositive and false-negative results of quantitative cultures must be accepted and this could have influenced the procalcitonin results.

In conclusion, the measurement of serum procalcitonin might be a reliable marker for the diagnosis of ventilator-associated pneumonia. Combining this marker with the simplified Clinical Pulmonary Infection Scores, a 100\% specificity was obtained and therefore this would avoid unnecessary antibiotic treatments by excluding false-positive diagnoses of ventilatorassociated pneumonia. However, the present study is a pilot study with several limitations and, despite the promising results, procalcitonin levels are not yet a tool to be used in routine practice to diagnose ventilator-associated pneumonia. The next step is the validation of the present results in an independent population representative of a medical intensive care unit.

\section{REFERENCES}

1 American Thoracic Society, Infectious Diseases Society of America. Guidelines for the management of adults with hospital-acquired, ventilator-associated, and healthcareassociated pneumonia. Am J Respir Crit Care Med 2005; 171: 388-416.

2 Chastre J, Fagon JY. Ventilator-associated pneumonia. Am J Respir Crit Care Med 2002; 165: 867-903.
3 Torres A, Carlet J. Ventilator-associated pneumonia. European Task Force on ventilator-associated pneumonia. Eur Respir J 2001; 17: 1034-1045.

4 Assicot M, Gendrel D, Carsin H, Raymond J, Guilbaud J, Bohuon C. High serum procalcitonin concentrations in patients with sepsis and infection. Lancet 1993; 341: 515-518.

5 Simon L, Gauvin F, Amre DK, Saint-Louis P, Lacroix J. Serum procalcitonin and C-reactive protein levels as markers of bacterial infection: a systematic review and meta-analysis. Clin Infect Dis 2004; 39: 206-217.

6 Christ-Crain M, Jaccard-Stolz D, Bingisser R, et al. Effect of procalcitonin-guided treatment on antibiotic use and outcome in lower respiratory tract infections: cluster-randomised, single-blinded intervention trial. Lancet 2004; 363: 600-607.

7 Hedlund J, Hansson LO. Procalcitonin and C-reactive protein levels in community-acquired pneumonia: correlation with etiology and prognosis. Infection 2000; 28: 68-73.

8 Brunkhorst FM, Al-Nawas B, Krummenauer F, Forycki ZF, Shah PM. Procalcitonin, C-reactive protein and APACHE II score for risk evaluation in patients with severe pneumonia. Clin Microbiol Infect 2002; 8: 93-100.

9 Brunkhorst FM, Eberhard OK, Brunkhorst R. Discrimination of infectious and noninfectious causes of early acute respiratory distress syndrome by procalcitonin. Crit Care Med 1999; 27: 2172-2176.

10 Oppert M, Reinicke A, Müller C, Barckow D, Frei U, Eckardt KU. Elevations in procalcitonin but not C-reactive protein are associated with pneumonia after cardiopulmonary resuscitation. Resuscitation 2002; 53: 167-170.

11 Duflo F, Debon R, Monneret G, Bienvenu J, Chassard D, Allaouchiche B. Alveolar and serum procalcitonin: diagnostic and prognostic value in ventilator-associated pneumonia. Anesthesiology 2002; 96: 74-79.

12 Gibot S, Cravoisy A, Levy B, Bene MC, Faure G, Bollaert PD. Soluble triggering receptor expressed on myeloid cells and the diagnosis of pneumonia. $N$ Engl J Med 2004; 350: 451-458.

13 el-Ebiary M, Soler N, Montón C, Torres A. Markers of ventilator-associated pneumonia. Clin Intensive Care 1995; 6: 121-126.

14 Knaus WA, Draper EA, Wagner DP, Zimmerman JE. APACHE II: a severity of disease classification system. Crit Care Med 1985; 13: 818-829.

15 Luna CM, Blanzaco D, Niederman MS, et al. Resolution of ventilator-associated pneumonia: prospective evaluation of the clinical infection scores as an early clinical predictor of outcome. Crit Care Med 2003; 31: 676-682.

16 Levy MM, Fink MP, Marshall JC, et al. 2001 SCCM/ ESICM/ACCP/ATS/SIS International Sepsis Definitions Conference. Crit Care Med 2003; 31: 1250-1256.

17 Garner JS, Jarvis WR, Emori TG, Horan TC, Hughes JM. CDC definitions for nosocomial infections, 1988. Am J Infect Control 1988; 16: 128-140.

18 Fàbregas N, Ewig S, Torres A, et al. Clinical diagnosis of ventilator-associated pneumonia revisited: comparative validation using immediate post mortem lung biopsies. Thorax 1999; 54: 867-873.

19 Meduri GU, Chastre J. The standardization of bronchoscopic techniques for ventilator-associated pneumonia. Chest 1992; 102: Suppl. 1, 557S-564S. 
20 Balows A, Hauslar WJ, eds. Manual of Clinical Microbiology. 5th Edn. Washington, DC, American Society of Microbiology, 1991.

21 Ioanas M, Ferrer M, Cavalcanti M, et al. Causes and predictors of nonresponse to treatment of the intensive care unit-acquired pneumonia. Crit Care Med 2004; 32: 938-945.

22 Póvoa P, Coelho L, Almeida E, et al. C-reactive protein as a marker of infection in critically ill patients. Clin Microbiol Infect 2005; 11: 101-108.
23 Póvoa P, Coelho L, Almeida E, et al. C-reactive protein as a marker of ventilator-associated pneumonia resolution: a pilot study. Eur Respir J 2005; 25: 804-812.

24 Luyt CE, Reynaud C, Combes A, et al. Usefulness of procalcitonin for the diagnosis of ventilator-associated pneumonia. Am J Respir Crit Care Med 2007; 175: A326.

25 Luyt CE, Guérin V, Combes A, et al. Procalcitonin kinetics as a prognostic marker of ventilator-associated pneumonia. Am J Respir Crit Care Med 2005; 171: 48-53. 